\title{
Efectos de los planes decenales de cultura en el fomento y proyección de las comunidades municipales. El caso de Piedecuesta (Colombia)
}

\section{Effects of the decennial plans of culture on the promotion and projection of municipal communities. The case of Piedecuesta (Colombia)}

\section{Luis Rubén Pérez Pinzón (iD}

Universidad Autónoma de Bucaramanga

1perez14@unab.edu.co

\begin{abstract}
Resumen
La cultura es un sector de la función pública dispuesto como campo de inversión e interés social por la Constitución Politica de Colombia, la ley de cultura (desde 1997) y el fomento de politicas y programas nacionales, regionales y locales por parte del Ministerio de Cultura como instancia superior de la gobernanza cultural colombiana. Los resultados de un proyecto de investigación concluido, a partir de una metodología cualitativa de carácter descriptivo, evidencian que los planes decenales de cultura han sido concebidos por las politicas culturales como la estrategia más eficaz para planificar las políticas públicas, organizar las inversiones, impactos y resultados de las creaciones y gestiones culturales con el apoyo de consejos municipales de cultura. En el caso de la ciudad de Piedecuesta, el plan de cultura es la mejor alternativa para garantizar la democracia participativa en la toma de decisiones y la apropiación de los productos culturales fomentados, financiados o estimulados con rentas públicas.
\end{abstract}

Palabras claves: cultura, politicas culturales, plan decenal, desarrollo local, Colombia.

\begin{abstract}
Culture is a sector of the public function arranged as a field of investment and social interest by the Political Constitution of Colombia, the Culture Law since 1997 and the promotion of national, regional and local policies and programs by the Ministry of Culture, as superior instance of Colombian cultural governance. The results of a completed research project, based on a descriptive qualitative methodology, show that the decennial plans of culture have been conceived by the cultural policies as the most effective strategy for planning public policies, organize investments, impacts and results of creations and cultural managements supported at the municipal councils of culture. In the case of the city of Piedecuesta, the plan of culture is the best alternative to ensure participatory democracy in decision-making and appropriation of cultural products fostered, financed or stimulated with public revenues.
\end{abstract}

Keywords: Culture, cultural plan, decennial plan, local development, Colombia

Articulo: Recibido el 24 de diciembre de 2018 y aprobado el 27 de junio de 2019.

\section{Cómo citar este articulo:}

Pérez Pinzón, LR (2019). Efectos de los planes decenales de cultura en el fomento y proyección de las comunidades municipales. El caso de Piedecuesta (Colombia). Reflexión Política 21(42), pp. XX. doi: 10.29375/01240781.3488 


\section{Introducción}

La cultura, al ser el conjunto de bienes, manifestaciones y expresiones de una nación, debe ser reconocida y protegida por el Estado por medio de políticas públicas y acciones de gobernanza público-privadas (Alcaldía Mayor, 2011). La Constitución de Colombia de 1991, acorde con las declaraciones internacionales firmadas por el Estado colombiano con la Organización de las Naciones Unidas (ONU), reconoció la cultura como un derecho y deber de sus ciudadanos, caracterizada por la diversidad y pluralidad. Por esta razón la carta política dispone el deber de proteger los bienes y manifestaciones culturales de la Nación (artículos 8, 63, 72 y 95) y, también, reconoce los derechos al fomento, acceso y protección de las creaciones culturales (artículos 61, 70 y 71).

Esta visión plural por conservar el patrimonio cultural heredado y promover el acceso a nuevas formas de expresión cultural adoptados se materializó en la Ley 397 General de Cultura de 1997, la cual fue modificada por la Ley 1185 de 2008 con el propósito de precisar que la cultura de los colombianos conformaba el "patrimonio cultural de la Nación” y que, consigo, debe ser responsabilidad de las autoridades y los ciudadanos de los ámbitos nacional, departamental, distrital, municipal o territorios étnicos, garantizar su “[...] salvaguardia, protección, recuperación, conservación, sostenibilidad y divulgación del mismo, con el propósito de que sirva de testimonio de la identidad cultural nacional, tanto en el presente como en el futuro" (Ley 1185, 2008, art. 4, par. b).

Las inversiones y planes nacionales promovidos desde el Ministerio de Cultura deben incorporarse a los planes de ordenamiento territorial, a partir de los planes especiales de manejo, protección, salvaguarda y divulgación de cada tipo patrimonial. Estos compromisos son reafirmados al ser adoptados como políticas públicas tanto en los planes cuatrienales de desarrollo, como en los planes decenales de cultura (Ley 1185, 2008, art. 7). Cada entidad territorial debe garantizar el cumplimiento de la Constitución y de la ley propiciando que: “[...] los planes de desarrollo de las entidades territoriales y los planes de las comunidades, grupos sociales y poblacionales incorporados a estos, deberán estar armonizados en materia cultural con el Plan Decenal de Cultura y con el Plan Nacional de Desarrollo y asignarán los recursos para la salvaguardia, conservación, recuperación, protección, sostenibilidad y divulgación del patrimonio cultural" (Ley 1185, 2008, art. 4, par. a).

En contraste con esa visión jerárquica y centralizada de la cultura desde las políticas ministeriales que regulan los planes especiales que deben cumplirse, los planes decenales de cultura, al considerar las tradiciones, necesidades, expectativas y proyecciones de las comunidades territoriales para las cuales son formulados, requieren de metas y objetivos que materializan y condicionan las metas de inversión de los planes de desarrollo de los gobernantes populares. Asimismo, delimitan los bienes, expresiones y manifestaciones que esas mismas comunidades consideran deben ser prioritarias para su fomento y promoción con ayuda de recursos municipales que permitan la construcción de ciudadanía, el fortalecimiento de la identidad y el sentido de pertenencia local, tanto en el sector urbano, como en el rural.

En el caso del municipio de Piedecuesta, su ubicación geoestratégica para la intercomunicación entre la costa Caribe y el interior de Colombia, así como de la frontera colombo-venezolana con el valle del Magdalena (Martínez \& Guerrero, 1995), ha propiciado crecientes y constantes procesos de inmigración desde provincias y departamentos limítrofes (ver imagen 1). Esto ha incidido en los cambios socioeconómicos y las prioridades de políticas culturales para atender las expectativas de los habitantes descendientes de familias piedecuestanas ancestrales (barrios del centro), las familias de desplazados e inmigrantes que llegaron a finales del siglo XX atraídas por las políticas en vivienda de interés social (barrios del extremo noroccidente y nororiente), así como la expansión urbanística de Bucaramanga al compensarse en Piedecuesta la falta de espacio para la oferta de vivienda residencial (barrios del extremo norte y sur) (Prada, 1997).

La ciudad de Piedecuesta, configurada por las desigualdades sociales y los conflictos políticos bipartidistas hasta la segunda década del siglo XX, sufrió una drástica transformación a partir de la década de 1980 a 1990 al promover la Constructora 
Hernández y Cía., diversos proyectos de vivienda de interés social para las familias de los obreros, empleados y comerciantes de Bucaramanga, así como para los campesinos desplazados por el conflicto armado de las poblaciones vecinas (Pérez, 2012a).

Piedecuesta, en un par de décadas, se transformó en una municipalidad receptora de inmigrantes, desplazados y emprendedores atraídos por la prosperidad de la capital del departamento de Santander (Valenzuela, 1995). Con este proceso, se han incrementado los índices de desempleo, improductividad e informalidad, lo cual se ha reflejado en que "según la percepción de sus habitantes, en materia de seguridad ciudadana, Piedecuesta es el municipio más inseguro del área con un 32 \%, le sigue Bucaramanga con 25 \%, Girón con 23 \% y Floridablanca con $18 \%$ \% (Carreño, 2018).

Durante las primeras décadas del siglo $\mathrm{XXI}$, la tendencia migratoria $\mathrm{y}$ urbanística ha conllevado a que Piedecuesta haya duplicado su población histórica; también se ha fragmentado su unidad urbana al aislarse el centro poblacional (con los barrios creados en el siglo XIX) de las urbanizaciones y ciudadelas en la periferia urbana al norte y sur de la ciudad (Pérez, 2012b). Estos, a su vez, se segregan de las zonas de invasión ilegal por desplazados y sintierra, así como de las zonas de desarrollo elitista caracterizadas por la construcción de condominios y ciudadelas para ciudadanos de estrato alto en los sectores rurales que, antiguamente, ocupaban haciendas con trapiches y cañaduzales (Pérez, 2012c).

Ese crecimiento descontrolado y desordenado ha conllevado al enfrentamiento sociocultural de los "piedecuestanos viejos" de los barrios antiguos con los residentes de las nuevas zonas de desarrollo urbano, quienes no manifiestan ningún interés por la identidad ni el desarrollo colectivo de la ciudad, a pesar de ser la población que, en las últimas elecciones de alcaldes municipales, congrega los electores mayoritarios y decisorios (Pérez, 2012d). Para unificar políticas públicas y lograr la participación activa de todos los sectores sociales del municipio, los alcaldes de Piedecuesta del siglo XXI han promovido, a través de sus planes de desarrollo, acciones participativas de los líderes comunales de los sectores urbanos y rurales para la planeación, desarrollo y ejecución de sectores fundamentales para el funcionamiento de la ciudad, como son la educación, la salud, el transporte, la seguridad, el desarrollo social de la población vulnerable y, en particular, de la cultura (Programa Gen, 2016).

La administración municipal del período 2012 - 2015 promovió y estableció las políticas públicas asociadas con el Plan Decenal de Turismo de Piedecuesta al constituirse en la industria de servicios con más proyección productiva ante la crisis de las exportaciones mineras del país (Alcaldía, 2016). Asimismo, la administración municipal del período 2016 - 2019, al reconocer la condición de ciudad intermedia de Piedecuesta, ha pretendido planificar su unidad colectiva en función del ejercicio de la cultura ciudadana. Para ello, es promovida la formulación e implementación del Plan Decenal de Cultura 2017-2026 (Alcaldía, 2017), con el apoyo del Consejo Municipal de Cultura.

Considerando las transformaciones legislativas culturales y la búsqueda de nuevos procesos de desarrollo local, los siguientes apartados tienen como objetivo principal describir los procesos de incorporación de las políticas culturales nacionales, considerando la promoción y formulación del Plan Decenal de Cultura de Piedecuesta. Este fue concebido como la estrategia más eficaz para planificar las políticas públicas, organizar las inversiones, impactos y resultados de las creaciones y gestiones culturales de los ciudadanos de Piedecuesta. También representa la mejor alternativa para garantizar la democracia participativa en la toma de decisiones y la apropiación de los productos culturales fomentados, financiados $o$ estimulados con rentas públicas.

Para tal fin, el texto responde a los siguientes interrogantes: ¿cómo el proceso de formulación del Plan Decenal de Cultura de Piedecuesta se apropió y dio cumplimiento a las políticas culturales de Colombia?, ¿de qué modo la planificación y proyección cultural adoptada a través de los planes decenales de cultura contribuye a delimitar y proyectar las necesidades, intereses e inversiones de las comunidades territoriales?

\section{Marco referencial: derechos culturales y consejos de cultura}

La Constitución Política de Colombia establece que el conjunto de ciudadanos que la conforman se rigen por un Estado social de derecho: la nación se rige por el reconocimiento de una república democrática, participativa y pluralista, asimismo, toda decisión que se adopte debe cumplir con el fin del Estado- 
nación. Según este, todas sus instituciones tienen la obligación de "facilitar la participación de todos en las decisiones que los afectan y en la vida económica, política, administrativa y cultural de la nación" (Procuraduría, 2018).

Esta visión sobre la toma de decisiones colectivas fundadas $\mathrm{y}$ justificadas únicamente en la participación democrática y plural de los ciudadanos fue reafirmada, en lo concerniente a los derechos culturales considerados "fundamentales" (Riveros, 2010), al decretarse en el artículo setenta que la promoción, fomento y acceso a la cultura de todos y para todos los colombianos debe planificarse y ejecutarse en igualdad de oportunidades, a través de procesos articulados a la educación permanente de carácter científico, técnico, artístico y profesional, con el propósito de recrear, crear o proyectar el proceso de creación de la identidad nacional entre los colombianos (Arjona, 2011). De tal manera, se requería reconocer la igualdad y dignidad de todas las que conviven en el país, así como considerar que cada uno de esos ciudadanos debían participar en los procesos de identificación, conservación, fundamentación y proyección de la cultura local, regional o nacional como fundamentos de la nacionalidad colombiana (Sanabria, 2010).

Sibien la Ley General de Cultura 097 de 1997 pretendió materializar el espíritu constitucional en lo concerniente al derecho a la cultura como una construcción colectiva desde una identidad diversa en instituciones, decretos y consejos consultivos, desafortunadamente continuaron primando las prácticas presupuestales y gubernamentales asociadas al fomento y financiación de las artes y los artistas más destacados o con quienes mejor cumplían con el ideal de "bellas artes" promovido desde mediados del siglo XIX de manera diferente en cada localidad y región de Colombia.

A esta dinámica se ha asociado el fomento colectivo de la cultura como parte de procesos institucionales de protección del patrimonio, la proyección de las identidades étnicas o el fomento de la investigación y la innovación, limitando toda acción o proyección cultural al "subdesarrollo jurídico” al que está condicionado y reducido el sector cultura (Uprimy, 2016). Es decir, las políticas culturales de cada municipio están limitadas a las inversiones y proyecciones de los planes de desarrollo de cada administración municipal y, así, de los planes de desarrollo departamental y nacional (Jaramillo, 2016). A falta de institutos descentralizados de cultura (e incluso existiendo esas organizaciones) las decisiones finales sobre financiación de proyectos en cultura son discrecionales del alcalde, previos acuerdos o solicitudes de los concejales municipales, sin importar las gestiones o recomendaciones de los consejeros de cultura (Alcaldía, 2017).

Ejemplo de ello son las limitadas tareas y funciones del Consejo Municipal de Cultura de Piedecuesta como máxima representación del sector cultural y artístico de todos los ciudadanos piedecuestanos, que se ve reducido a la condición de órgano consultivo y de asesoría de la Alcaldía Municipal, cuya autoridad e importancia es desconocida o menospreciada por el Concejo Municipal (ayuntamiento) y los demás consejos operativos con los que cuenta el municipio para la definición de su política pública. Con ello, los representantes de los gremios y sectores articulados por el Consejo son condicionados por los cambios electorales de alcaldes y concejales y, posteriormente, por las decisiones y relevancia que tienen sus recomendaciones ya que no cuenta con presupuesto, funciones ni planes de acción a largo plazo.

Las funciones del Consejo de Cultura de Piedecuesta son acordes con las disposiciones de la Ley de Cultura (Ley 97, 1997), pero su actuar se limita a sugerir o evaluar las decisiones gubernamentales al ser concebido para:

1. Contribuir al mejoramiento de la calidad de vida de las comunidades, animando y apoyando procesos de convivencia social democrática que impulsen su desarrollo y el entendimiento intercultural.

2. Asesorar al gobierno local en la formulación y ejecución de las políticas culturales, el diseño, la implementación y evaluación de la planificación del desarrollo municipal, teniendo en cuenta la dimensión cultural y socializar su actuación con la comunidad.

3. Articular los procesos y actividades relacionados con el fomento, promoción y difusión del patrimonio cultural y artístico del municipio de Piedecuesta, estimulando y apoyando la organización y fortalecimiento del sector.

4. Vincular y articular al nivel municipal los actores y espacios de concertación de los niveles departamental, regional y nacional y los demás sistemas involucrados en la planificación y 
ejecución de procesos culturales.

5. Contribuir a la estructuración de redes de información y comunicación que posibiliten a los pobladores el acceso a los bienes y servicios culturales de manera oportuna.

6. Estimular el desarrollo cultural de las comunidades del municipio.

7. Actuar como entes articuladores de las actividades relacionadas con el fomento, la promoción y la difusión de las actividades artísticas y culturales programadas en el municipio.

8. Promover y hacer recomendaciones que sean pertinentes para la formulación, cumplimiento y evaluación de los planes, programas y proyectos culturales.

9. Participar en las labores de los órganos de planeación, presentando propuestas para la formulación o ajustes de los planes de desarrollo que vinculen la dimensión cultural al desarrollo del municipio.

10.Apoyar y asesorar al alcalde municipal en el diseño e implementación de las políticas culturales del municipio, teniendo en cuenta las políticas departamentales y/o nacionales.

11.Establecer su propio reglamento.

12. Elaborar su plan de trabajo y rendir informes de avance en la gestión.

13.Liderar la formulación y firma de pactos ciudadanos por la cultura con los aspirantes a la alcaldía del municipio.

14. Participar en la elección del representante del Consejo Municipal al Consejo Departamental de Cultura.

15. Participar en la elección del representante al Consejo Territorial de Planeación (Acuerdo 14, 2013).

Surgió así, en 2015, la necesidad de una política pública para el sector cultura de Piedecuesta que, anteponiendo los procesos de elección de legisladores y gobernantes municipales, así como las inversiones cuatrienales delimitadas por el Plan de Gobierno y/o el Plan de Desarrollo de cada alcalde, logre delimitar las metas, recursos y resultados esperados durante varios períodos gubernamentales y, asimismo, rescatar las visiones y proyecciones adoptadas en el primer intento de planificación cultural municipal en 2001. De allí que todo plan decenal de cultura sea "[...] una ruta. Un acuerdo. Una construcción colectiva. Una forma de interrogar el futuro común... Una apuesta dirigida hacia la construcción de una ciudadanía democrática cultural. Una ciudadanía con el poder de expresarse sin temor y en comunión con los otros" (Mincultura, 2001, p. 9).

De acuerdo a los postulados de la planificación del desarrollo dispuestos por la Ley 152 de 1994, los planes de cultura para el fomento estratégico del desarrollo local son considerados además “[...] la mejor herramienta a la hora de gestionar y administrar un proceso, espacio, proyecto o servicio cultural, pues permite diseñar, planificar e implementar las acciones necesarias para darle vida y potenciar su desarrollo en el tiempo". Al no ser lo mismo que una programación artística o un emprendimiento cultural, cada plan de cultura "[...] no debe realizarse ajeno a las personas que resultarán beneficiarias de las intervenciones programadas. Son ellas quienes viven las problemáticas $\mathrm{y}$, por lo tanto, son quienes con mayor claridad pueden hablar de sus causas, efectos y posibles soluciones, como también de las acciones de mayor pertinencia" (Jaramillo, 2016, pp. 1 y 3).

\section{Metodologia: construcción plural y participativa de planes en cultura}

A partir de un trabajo de investigación con enfoque cualitativo, de carácter descriptivo y orientado a la identificación de necesidades y expectativas de comunidades sociales y artísticas específicas por medio de entrevistas, encuentros, grupos focales y eventos públicos de socialización, se demostró a la alcaldía de Piedecuesta la necesidad de formular el Plan Decenal de Cultura para Piedecuesta, aunado a la importancia de cumplir con la primera meta cultural del Plan de Desarrollo Municipal 20162019 asociada con la adopción de dicho Plan.

En el informe final se planteó que la visión cultural para Piedecuesta, desde 2017, estaba inspirada en el lema "construir cultura es construir ciudad y ciudadanía”, lo cual requiere que los ciudadanos que hacen parte tanto de la ciudad como del municipio se integren al proceso de construcción de las políticas culturales que guiarán las acciones e inversiones culturales entre 
los piedecuestanos hasta 2026 (Alcaldia, 2017).

Siguiendo la experiencia metodológica del Plan de Desarrollo Cultural de Cultura de Medellín (Acuerdo 49, 2016), el proceso seguido requirió considerar y sistematizar las opiniones de los creadores, gestores, artistas y organizaciones artísticas de Piedecuesta, así como las necesidades y expectativas de los líderes comunales de cada barrio y vereda, en especial, las recomendaciones de los ciudadanos que conforman los comités y organizaciones culturales al interior de cada Junta de Acción Comunal. La formulación de las políticas culturales para el municipio de Piedecuesta partió de un diagnóstico del sector cultura por parte del equipo técnico e interdisciplinario de investigadores a partir de los saberes y perspectivas de cada uno de los representantes de los campos o subsectores culturales que integran el Consejo Municipal de Cultura, siguiendo, así, los procedimientos adoptados por otros equipos universitarios interdisciplinarios para la formulación de los planes decenales de cultura de las principales ciudades y departamentos del país (Departamento, 2015).

A la par de la identificación de las dificultades, fortalezas y proyecciones decenales de cada subsector, se compiló y organizó la información documental oficial proveniente de los planes de desarrollo de administraciones anteriores, el Concejo Municipal, la secretaría de desarrollo social y el Consejo Municipal de Cultura, etc. Los resultados de esa etapa fueron la delimitación, en asocio a los profesionales en gestión cultural de la Alcaldía, de los fines, enfoques, campos y dimensiones que guiaron la construcción colectiva del plan decenal.

La siguiente etapa del Plan de Piedecuesta, acorde al Plan propuesto por la Alcaldía de Medellín (2011), consistió en articular la planeación estratégica acordada a las decisiones, políticas, prácticas, propuestas y tradiciones procedimentales en la gestión y administración del sector cultura de Piedecuesta, a través de una propuesta preliminar del plan. Esta fue presentada al Consejo Municipal de Cultura, reunido en pleno con el propósito de reafirmar la estructura del plan: los principios, líneas y proyecciones estratégicas propuestos para los siguientes diez años. Asimismo, se pidió a los consejeros organizar y dar prioridad a las líneas que consideraban más importantes y necesarias de ejecutar en la próxima década, siendo las primeras seis opciones asociadas con las políticas a cumplir a corto plazo durante el siguiente trienio.
Delimitadas las políticas culturales que debían orientar un punto de partida para la construcción colectiva del plan decenal de cultura (Alcaldía Mayor, 2012), se procedió a hacer la convocatoria pública de mesas de trabajo sectoriales para todos los creadores, gestores, gremios organizaciones e instituciones culturales de Piedecuesta con representación y reconocimiento ante el Consejo Municipal de Cultura. Sus líderes y representantes gremiales se dieron cita en el Centro Cultural de Piedecuesta durante tres fines de semana para leer, discutir y mejorar cada una de las líneas y metas proyectadas.

Al lograrse la concertación del plan con los artistas fueron convocados todos los representantes y comités de cultura de cada una de las juntas de acción comunal urbanas, con el fin de socializar y mejorar la versión inicial del plan a partir de sus saberes, expectativas y necesidades, problemas y "meta-problemas" (Departamento, 2015), durante las mañanas de los sábados de noviembre. Asimismo, se convocaron todas las juntas de acción comunal rurales a participar del proceso, siendo visitadas y congregadas en los cuatro extremos rurales de Piedecuesta (Instituto Holanda, Instituto Valle del Río de Oro, Instituto del Oriente y Centro Cultural) en las tardes de los días sábado y domingo, por ser los días no laborales más factibles para efectuar la reunión.

El plan mejorado y ampliado como resultado de la concertación y revisión de los subsectores culturales y los representantes comunales fue divulgado a través de la página web de la alcaldía de Piedecuesta y los correos electrónicos de los cultores, consejeros y líderes comunales que se hicieron presentes a las mesas de trabajo (Alcaldía, 2017). Con esto, el proceso inicial de consulta y diagnóstico entre los gremios y las comunidades del municipio requería concluir con una consulta, divulgación y búsqueda exhaustivas de acuerdos entre esos mismos gremios al ser publicados -a través de diferentes soportes y medios de comunicación- los informes, textos y proyecto de acuerdo municipal (Díaz, 2015).

La versión final acordada entre la Universidad Autónoma de Bucaramanga y la Alcaldía Municipal de Piedecuesta como parte del proyecto de investigación E67010 fue presentada a la administración municipal en el informe final de investigación del Plan Decenal de Cultura, acompañada de una propuesta de Proyecto de 
Acuerdo municipal para la evaluación, debate y aprobación por los honorables miembros del Concejo Municipal de Piedecuesta.

Habiendo previamente revisado $\mathrm{y}$ aprobados los documentos por el asesor de cultura, la Secretaría de Desarrollo Social, el alcalde municipal y el secretario jurídico del municipio, se constituyó el Consejo Municipal de Cultura en veedor y evaluador de su ejecución (Alcaldía, 2017). Sin embargo, al igual que la experiencia del Plan Decenal de Medellín acordado en 2011, pero tan solo adoptado a finales de 2015 (Acuerdo 49, 2015), el Plan para Piedecuesta, después de dos años de existencia, aún no ha logrado ser puesto en discusión, ajuste y adopción por las autoridades legislativas del Municipio.

\section{Resultados: problemas, expectativas y proyecciones culturales para Piedecuesta}

Cada Plan Decenal de Cultura es un instrumento de planificación de las inversiones públicas y los productos e impactos que el sector cultura tendrá en cada territorio municipal de Colombia a corto plazo (administración municipal actual), mediano (administraciones municipales siguientes) y largo (programas y proyectos que se deben continuar, fortalecer o sustituir en el siguiente Plan) (Alcaldía Mayor, 2012).

Al ser acordadas y promovidas las políticas culturales municipales, los alcaldes y concejales de diferentes administraciones pueden justificar las inversiones públicas en cultura durante la siguiente década, haciendo permanente y activo seguimiento a los indicadores, productos y gastos que se requieren para cumplir con los objetivos, lineamientos y metas culturales visionadas para el mejoramiento de la calidad de vida y la participación ciudadana de todos los ciudadanos (Díaz, 2015). Para tal fin, son asesorados por el Consejo Municipal de Cultura y los funcionarios encargados del sector cultura, que también respaldan sus decisiones en la veeduría ciudadana ejercida por las instituciones culturales, las agremiaciones de artistas y los gremios socioeconómicos del municipio (Alcaldía, 2017).

Para la formulación e implementación del Plan Decenal de Cultura, los creadores, gestores y organizaciones artísticas de Piedecuesta se han constituido en los referentes culturales de mayor reconocimiento y dinamismo, al ser distinguidos de forma individual o colectiva por sus acciones de creación y promoción cultural en artes plásticas, artes audiovisuales, danza, teatro, música, letras y artesanos, de acuerdo a los gremios reconocidos para elegir representantes ante el Consejo Municipal de Cultura (Acuerdo 14, 2018).

En cuanto a formación e instrucción en las diferentes disciplinas de creación y promoción cultural, los creadores de Piedecuesta coinciden en la necesidad de capacitarse en gestión, administración y ejecución de proyectos. Sobre este punto, el sector de literatura y el de teatro coinciden en manifestar que la educación formal no es prioritaria para el desarrollo de sus trabajos $\mathrm{y}$ no hace parte de las necesidades del gremio a corto plazo. La formación debería ser no formal y partiendo del intercambio de experiencias a nivel nacional e internacional (Alcaldía, 2017).

Los siete campos de creación y promoción cultural tienen una larga trayectoria. Es de resaltar la banda de músicos, con más de 100 años de haber sido constituida, cuya influencia se evidencia en los diferentes procesos artísticos que han marcado la historia cultural del municipio de Piedecuesta (Prada, 1995). Sectores como la música, el teatro, la danza y las artes plásticas aparecen de forma visible en diferentes escenarios, con sus presentaciones y participación en espacios públicos. Por su parte, la literatura, la artesanía y el sector audiovisual cuentan con menor reconocimiento e inclusión, por falta de eventos y espacios específicos de divulgación y promoción.

Para los creadores y gestores artísticos, celebraciones como la Semana Santa y la Semana de la Piedecuestaneidad se constituyen en los eventos culturales más importantes de Piedecuesta, al ser convocados y participar la mayoría de los sectores culturales. En contraste con estos eventos de primera importancia para las administraciones, los creadores y gestores independientes afirmaron no contar con apoyo ni estímulos para el desarrollo de sus sectores por parte de la administración municipal, reduciéndose sus ingresos a los aportes obtenidos del Ministerio de Cultura, a la Cámara de Comercio y a la Gobernación, con las becas y estímulos que periódicamente otorgan (Alcaldía, 2017).

Uno de los principales hallazgos diagnósticos del plan fue que todos los sectores artísticos tienen una proyección a futuro, no delimitada, pero sí diseñada para el fortalecimiento de cada uno de ellos de forma individual. No existe un trabajo articulado 
entre sectores, ni una visión colectiva de las artes en el municipio de Piedecuesta. Solo la danza maneja una propuesta colectiva de trabajo, proyección y gestión de recursos, mientras que los demás campos de la cultura trabajan (de forma individual o grupal) como organizaciones independientes. De tal modo, los representantes de los diferentes sectores culturales del municipio, desde su condición de integrantes del Consejo Municipal de Cultura, manifestaban no conocer a profundidad el componente cultura del Plan de Desarrollo Municipal 2016-2019, al no sentirse plenamente incluidos en el mismo (Alcaldía, 2016, p. 71).

Los procesos de investigación están presentes en el desarrollo artístico de todos los sectores, particularmente del sector literatura y del sector teatro, que tienen la investigación como esencia fundamental de su trabajo. Sin embargo, se requieren estudios interinstitucionales que hagan un inventario de los procesos artísticos y creativos que se llevan a cabo en Piedecuesta, al no existir estímulos, reconocimientos y fomento a los creadores y gestores con más trayectoria. Entre las necesidades más apremiantes para los creadores y gestores culturales independientes está la creación de una escuela de artes y oficios que fortalezca el Instituto de Bellas Artes y les permita unirse y brindar al municipio una oferta cultural de calidad, con un espacio físico para su desarrollo (Alcaldía, 2016, p. 72).

Tal carencia de escenarios especializados ha conllevado a una petición unánime por rescatar y habilitar el Centro Cultural del municipio para los fines culturales y artísticos para los que fue creado. Actualmente, fue adecuado como edificio para las oficinas administrativas que requiere la alcaldía municipal (Prada, 2015). Solo con espacios exclusivos para la promoción y divulgación de las creaciones culturales locales se logrará atraer públicos y gestionar eventos que contribuyan al desarrollo de las actividades artísticas, culturales y patrimoniales que anhelan los piedecuestanos de todas las edades y sectores (Alcaldía, 2017).

Desde la perspectiva de los líderes comunales rurales, en especial de los integrantes de los comités culturales que conforman cada Junta de Acción Comunal Veredal, la participación campesina no siempre es activa, pues se reduce a la asistencia durante las ferias ganaderas y a ser consumidores culturales de los festejos públicos masivos, desde que se celebra la fiesta del campesino y el día del Corpus como parte del festival piedecuestano (Alcaldía, 2017). Durante ese día se ha logrado una integración más comprometida y directa de las comunidades campesinas, al presentar a concurso público los arcos adornados con los mejores productos agrícolas y artesanales de la tierra, así como al atender y responder a la curiosidad agrícola, ecológica y etnológica de los visitantes del evento (Pérez, 2012d).

En cuanto a prácticas artísticas específicas, las danzas tradicionales se han perdido, ya no se practican. Esto se refleja en la inexistencia de grupos $\mathrm{u}$ organizaciones campesinas que promuevan la danza durante ocasiones festivas o eventos culturales (Valenzuela, 1995). La poca actividad relacionada con bailes tradicionales se evidencia ya en su falta de promoción en las escuelas y colegios rurales y, asimismo, no se considera necesaria ni atractiva en la vida cotidiana ni para la participación en los festivales, eventos o actividades culturales que se programan en el sector urbano. De allí la importancia de divulgar y promover la danza del chulo como baile típico municipal desde 2015 y como referente cultural del departamento (Alcaldía, 2017).

De modo contrario a la danza, la música tradicional campesina (rumba criolla, alegre o carranguera) tiene mayor presencia y reconocimiento rural y urbano. Existen algunos grupos de música en cada extremo veredal del municipio, aunque la participación pública y continúa de los mismos es limitada por la falta de espacios y eventos de interés masivo, además del desinterés de los sellos musicales y las estaciones radiales por divulgarla (Prada, 1995).

Según los líderes comunales veredales, la pérdida de talentos musicales rurales está asociada a la falta de apoyo, formación, donación de instrumentos musicales y divulgación de las expresiones artísticas campesinas, que se ven menospreciadas o cuestionadas en su propio entorno. Problemática aunada a la falta de atención gubernamental a los gestores culturales que promueven acciones de formación artística con los niños y niñas campesinas para orientar su uso del tiempo libre y prevenir problemas del entorno campesino como la drogadicción y la delincuencia generada por el microtráfico, el ocio y la deserción escolar (Alcaldía, 2016). Para los líderes rurales del sur y occidente de Piedecuesta, es preocupante la creciente pérdida de espacios, manifestaciones y expresiones artísticas asociadas con el mundo rural, como la danza y la música andina, razón por la cual 
es unánime la solicitud de proyectos y programas, públicos o particulares, que recuperen y garanticen la continuidad de sus tradiciones (Alcaldía, 2017).

El olvido y desprecio de la cultura popular campesina se evidencia de forma más preocupante en lo concerniente a la tradición oral, al olvidarse o menospreciarse los cuentos, leyendas, mitos, dichos y refranes, etc., de la ruralidad. Por tal motivo, algunos gestores culturales comunales sugirieron la pronta recuperación de ese patrimonio cultural intangible por medio de compilaciones que estén disponibles en las bibliotecas, asimismo, crear archivos audiovisuales con los testimonios de las personas ancianas que aún custodien y promuevan esos conocimientos culturales (Alcaldía, 2000). No obstante, algunas creencias y convicciones propias de las tradiciones orales y las prácticas cotidianas tienden a conservarse ante la ausencia de toda forma de atención hospitalaria o servicios médicos. Por ejemplo, en los sitios rurales más distantes de Piedecuesta, los campesinos aún recurren a las prácticas de curación con ayuda de sobanderos y al uso frecuente de plantas medicinales, al heredar de sus ancestros conocimientos populares sobre sus propiedades curativas (Alcaldía, 2017).

Estos conocimientos están en peligro de extinción cultural ante la escasez de agua que impide o extingue las plantas medicinales silvestres prexistentes, ante el fallecimiento de adultos mayores que no transmitieron sus saberes botánicos y homeopáticos a sus vecinos y herederos, así como por la ignorancia o desprecio de los adultos jóvenes y adolescentes hacia las creencias populares (Programa Gen, 2016). De allî la importancia de reposicionar en el contexto social a los ancianos como herederos y portadores de los saberes populares para recuperar y fortalecer el aprovechamiento de las plantas medicinales de la región, gestionar capacitaciones y manuales de uso público para conocer las propiedades de las plantas, sus usos y las dosis recomendadas para ser empleadas (Alcaldía, 2017).

\subsection{Políticas y estrategias culturales del 2017 al 2026}

La interacción de las visiones y sugerencias de los creadores y gestores urbanos con las necesidades y expectativas de los líderes comunales rurales permitieron formular un Plan Decenal de Cultura compuesto por veinte políticas culturales para ser desarrolladas entre 2017 y 2026. Estas se subdividen, a su vez, en sesenta y seis metas estratégicas (Alcaldía, 2017).

De estas políticas y metas, las más discutidas y reclamadas en las mesas de trabajo para la concertación de los documentos finales fueron las asociadas con la línea estratégica veinte. Mediante ellas se buscaba regular desde el sector cultura todos aquellos impactos económicos $\mathrm{y}$ productivos, en desarrollo urbano o uso del espacio público y la existencia de infraestructura, movilidad y conectividad que garantizaban (o al mismo tiempo limitaban) el desarrollo artístico, la preservación del patrimonio y la convivencia social de los ciudadanos de la ciudad de Piedecuesta.

Entre esas estrategias, como se mencionó, es de destacar la vigésima, mediante la cual se dispone que, hasta 2026:

creadores, gestores, consejeros, funcionarios $\mathrm{y}$ promotores culturales de Piedecuesta contribuy[a] $\mathrm{n}$ al fomento del cuidado y uso racional de los recursos naturales, la sostenibilidad territorial y el acceso a los espacios y servicios públicos a partir de las iniciativas, actividades, eventos, convocatorias, etc., de los demás sectores socioculturales del municipio. (Alcaldía, 2017, p. 80)

Para cumplir esta línea estratégica directamente asociada con el desarrollo sostenible y sustentable para la ciudad, se plantearon acciones específicas de carácter educativo para:

[...] la incorporación de componentes formativos explícitos que contribuyan al cuidado y uso racional de los recursos naturales entre todos los sectores socioculturales del municipio en cada una de las creaciones, proyectos, programas, actividades, eventos, etc., financiados anualmente con dineros públicos. (Alcaldía, 2017, p. 99)

Estas, complementadas por acciones de carácter científico desde

[...] convenios de capacitación, fortalecimiento y transferencia de la cultura científica con las instituciones, organizaciones, empresas y emprendedores cuyos centros de desarrollo, innovación o producción se encuentran en el parque tecnológico y las zonas de uso industrial o de investigación científica en el territorio piedecuestano. (Alcaldía, 2017, p. 101) 
Incluso, acciones de carácter comunicacional masivo al garantizarse:

[...] el acceso y conectividad de los piedecuestanos a las ofertas y propuestas culturales promovidas a través del espectro electromagnético empleado por las estaciones radiales y los canales de televisión, así como a través del acceso gratuito a conexiones alámbricas e inalámbricas de contenidos en línea y la divulgación de un portal cultural digital a través de redes sociales. (Alcaldía, 2017, p. 99-102)

El desarrollo sostenible también requerirá la participación activa de los gestores culturales al adoptarse "[...] las políticas de crecimiento y desarrollo mediante las cuales se planifique como parte de las nuevas construcciones urbanas y rurales la existencia de salones comunales, centros culturales, parques recreacionales, sitios de congregación y eventos” (Alcaldía, 2017, p. 99); “[...] la formación en cultura ciudadana, valores cívicos, uso del espacio público, características y dimensiones de la piedecuestaneidad, etc.” (Alcaldía, 2017, p. 100) y, “[...] para la toma de decisiones en lo concerniente a las políticas territoriales, procesos de urbanismo, planes ambientales, proyectos de uso o ampliación del espacio público y demás aspectos asociados con los derechos ambientales y colectivos de los ciudadanos piedecuestanos" (Alcaldía, 2017, p. 100).

El resultado final esperado de esos esfuerzos es la apropiación artística y el disfrute cultural de los espacios y recursos públicos, para lo cual se espera que el municipio de Piedecuesta promueva anualmente:

[...] acciones culturales que propicien una convivencia de proximidad y coexistencia entre ciudadanos, al reunirse en los espacios públicos para disfrutar de los festivales municipales, las presentaciones periódicas de creadores $\mathrm{u}$ organizaciones financiadas por la administración municipal, la asistencia a exposiciones, encuentros y capacitaciones itinerantes, etc. (Alcaldía, 2017, p. 100).

Por otra parte, se pretende lograr que el Consejo Municipal de Cultura se constituya, después de una década de gestiones y creaciones colectivas, en un estamento de gestión y promoción cultural transversal, cuyas recomendaciones y propuestas sean consideradas por todas las dependencias municipales. Para ello:
Los gestores y creadores culturales, líderes comunales, veedores ciudadanos, representantes gremiales e institucionales, etc. participarán activamente en la consolidación de las políticas culturales dispuestas por Acuerdo municipal al acompañar periódicamente a los integrantes del Consejo Municipal de Cultura en los procesos de formulación de recomendaciones comunitarias para el manejo de los recursos municipales del sector cultura, el seguimiento público a los apoyos y estímulos otorgados a través del banco de proyectos elegibles, la articulación de las minorías étnicas y los comités comunales en cultura y recreación a los proyectos y programas municipales, así como la evaluación y control ciudadano a la gestión de los funcionarios encargados del cumplimiento de las estrategias y metas asociadas con las ocho dimensiones del Plan Decenal de Cultura del Municipio de Piedecuesta 2017 - 2026. (Alcaldía, 2017, pp. 101-102).

\section{Conclusiones}

Ciudades históricas como Piedecuesta, adscrita al departamento de Santander en la República de Colombia, cuentan con una identidad, tradición y patrimonio culturales que, gradualmente, han sido transformados o adaptados por parte de la población tradicional ante los crecientes y cambiantes procesos de inmigración de habitantes provenientes de otras provincias, regiones y países. Migrantes que durante los primeros años de permanencia en la ciudad tienden a considerarse ignorantes, extraños o desconocidos por los bienes, tradiciones y manifestaciones que han dado identidad cultural a los piedecuestanos por más de doscientos años.

La adopción de políticas públicas reguladas por mecanismos legales como el Plan Decenal de Cultura, dispuesto por la Ley General de Cultura de 1997, contribuye al reencuentro de los ciudadanos con la memoria, tradición e identidad promovidas por los creadores y cultores culturales, asimismo, reconoce las necesidades, expectativas e intereses de los ciudadanos que garantizan la vitalidad del patrimonio cultural.

La materialización de esa pluralidad de visiones y expectativas requiere del trabajo articulado y moderado por una instancia de representación, reconocimiento y legitimidad ante todos los gremios, sectores y comunidades, como es el caso del Consejo Municipal de Cultura, a partir 
del cual, en Piedecuesta, se logró la concertación del plan preliminar entre los artistas que hicieron parte del proceso y la presentación y mejoramiento de una propuesta común ante los representantes y comités de cultura de cada una de las juntas de acción comunal urbanas y rurales del municipio. Finalmente, la versión final del Plan, resultado de la concertación y revisión de los subsectores culturales y los representantes comunales, fue divulgada a través de la página web de la Alcaldía de Piedecuesta y los correos electrónicos de los cultores, consejeros y líderes comunales que se hicieron presentes en las mesas de trabajo con el propósito de legalizar su aprobación por parte del Concejo Municipal.

Los integrantes del Consejo Municipal de Cultura de Piedecuesta son los encargados de gestionar e implementar las politicas y metas culturales acordadas en el Plan Decenal de Cultura adoptado de 2017 a 2026. Sin embargo, las acciones culturales deben involucrar a todos los gremios del sector cultura y a todas las comunidades socioeconómicas del municipio para garantizar el impacto y eficacia de las inversiones públicas, así como para contribuir a la articulación de los residentes foráneos a las prácticas y costumbres de los piedecuestanos nativos.

En el caso de Piedecuesta, la formulación de un plan de cultura comprometido con el desarrollo sostenible evidenció, en lo concerniente a su línea estratégica final, que la importancia de la intermediación crítica del sector cultura en la toma de decisiones, así como la articulación de las organizaciones académicas, científicas y comunicacionales, debe conllevar a acuerdos y acciones colectivas de desarrollo local asociadas con la preservación y priorización de los recursos naturales, el desarrollo urbanístico concertado, la formación en cultura ciudadana, el manejo del espacio público, la financiación pública de eventos colectivos y la investigación científica en los parques tecnológicos del municipio.

Es de interés prioritario la consolidación de las políticas culturales dispuestas por acuerdos municipales que acompañen periódicamente a los integrantes del Consejo Municipal de Cultura en cada uno de los procesos de formulación de recomendaciones comunitarias para el manejo de los recursos municipales del sector cultura. Estas deben ser acordes a las ocho dimensiones del Plan Decenal de Cultura del Municipio de Piedecuesta que deben ser adoptadas por las administraciones municipales y sus planes de desarrollo desde 2017 hasta 2026.
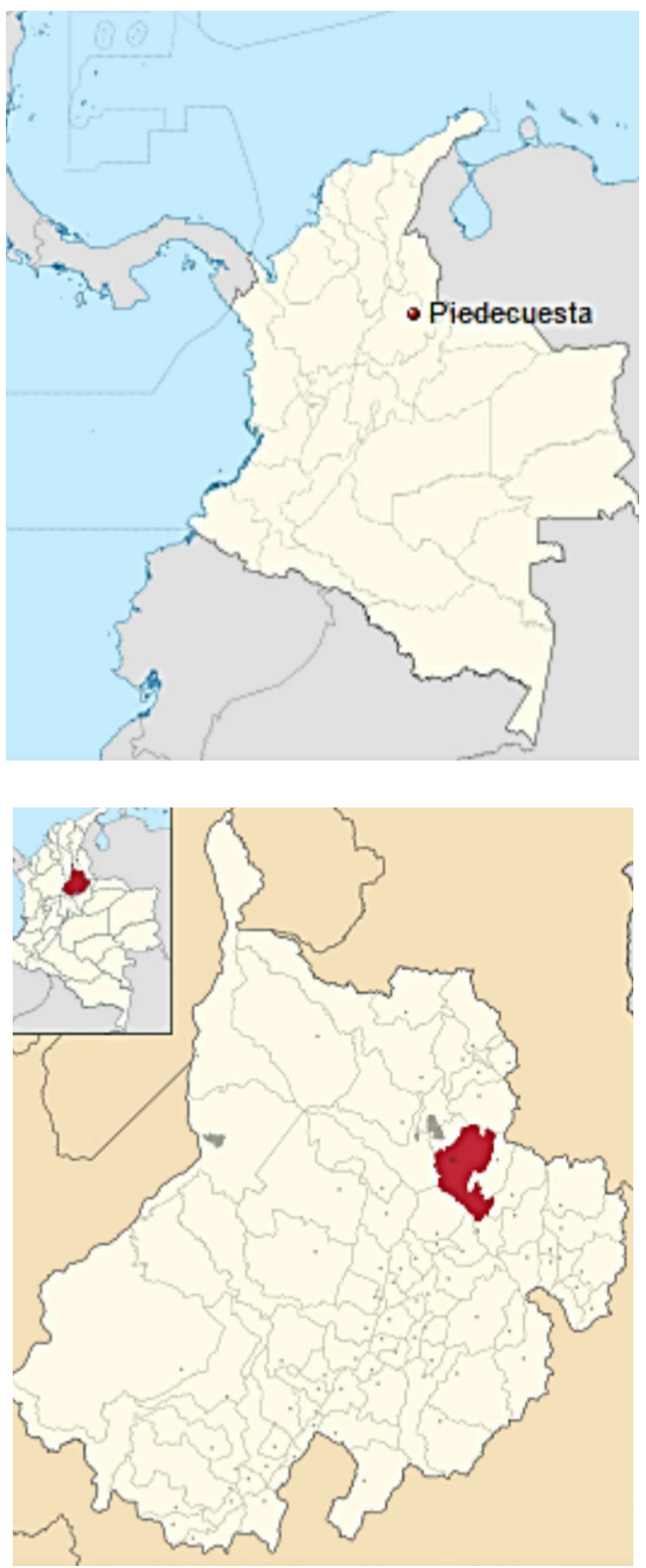

Figura 1. Ubicación geográfica del municipio de Piedecuesta

Fuente: Alcaldía de Piedecuesta (2017).

\section{Referencias}

Alcaldía Mayor de Bogotá (2011). Constitución de 1991 y cultura: Bogotá: Alcaldía Mayor - Corporación Opción Legal.

Alcaldía Mayor de Bogotá (2012). Plan Decenal de Cultura Bogotá D. C. 2012-2021. Bogotá: Secretaría de Cultura. Recreación y Deporte - Universidad 
Nacional de Colombia.

Alcaldía Municipal de Medellín (2011). Plan de Desarrollo Cultural de Medellín 2011-2020. Medellín: Alcaldía Municipal de Medellín.

Alcaldía Municipal de Piedecuesta (2000). Proyecto "Piedecuesta recupera su memoria”-barrios y veredas de Piedecuesta. Piedecuesta: Alcaldía Municipal de Piedecuesta.

Alcaldía Municipal de Piedecuesta (2016). Plan de Desarrollo Municipal 2016-2019: Piedecuesta mi plan. Piedecuesta: Alcaldía Municipal de Piedecuesta.

Alcaldía Municipal de Piedecuesta (2017). Plan Decenal de Cultura de Piedecuesta 2017-2026: Construir cultura es construir ciudad y ciudadanía. Piedecuesta: Alcaldía Municipal de Piedecuesta - Universidad Autónoma de Bucaramanga - Luis Rubén Pérez Pinzón.

Arjona, G. (2011). Derechos culturales en el mundo, Colombia y Bogotá. Bogotá. Secretaría Distrital de Cultura, Recreación y Deportes.

Carreño, Y. (2018). Piedecuesta, el municipio más inseguro del área metropolitana de Bucaramanga, según el Cómo Vamos. Periódico 15. Disponible en https://bit.ly/2LuzYEx

Concejo Municipal de Piedecuesta (2013). Acuerdo 14 de 2013. Gaceta Oficial del Municipio de Piedecuesta. Disponible en http://concejodepiedecuesta.org/acuerdo-no014-2013/

Concejo Municipal de Medellín (2015). Acuerdo 49 de 2015. Gaceta Oficial del Municipio de Medellín: XXII(4356), 13.

Congreso de la República de Colombia (1997). Ley 97 de 1997. D.O.: 43102 (1): 1

Congreso de la República de Colombia (2008). Ley 1185 de 2008. D.O.: 46929 (1): 1

Constitución Política de Colombia (1991). Disponible en: https://goo.gl/DecyU6

Departamento de Cundinamarca (2015). Plan Decenal de Cultura del Departamento de Cundinamarca 2016-2026. Bogotá: Instituto de Cultura y Turismo de Cundinamarca - Universidad Externado de Colombia.

Díaz, M. (2015). Pensar el plan de desarrollo cultural de Medellín: una experiencia de articulación de actores (tesis de maestría). Universidad Eafit, Medellín.
Jaramillo, A. (2016). Plan Municipal de Cultura. Medellín: Secretaría de Cultura de la Gobernación de Antioquia.

Martínez, A. \& Guerrero, A. (1995). La provincia de Soto: orígenes de sus poblamientos urbanos. Bucaramanga: Escuela de Historia UIS.

Ministerio de Cultura de Colombia (Mincultura) (2001). Plan Nacional de Cultura 2001-2010. Bogotá: Consejo Nacional de Cultura.

Pérez, L. (2012a). Ciencias naturales de Piedecuesta. Bucaramanga: Universidad Autónoma de Bucaramanga - Luis Rubén Pérez Pinzón.

Pérez, L. (2012b). Ciencias sociales de Piedecuesta. Bucaramanga: Universidad Autónoma de Bucaramanga - Luis Rubén Pérez Pinzón.

Pérez, L. (2012c). Ciencias económicas de Piedecuesta. Bucaramanga: Universidad Autónoma de Bucaramanga - Luis Rubén Pérez Pinzón.

Pérez L. (2012d). Ciencias religiosas de Piedecuesta. Bucaramanga: Universidad Autónoma de Bucaramanga - Luis Rubén Pérez Pinzón.

Pérez Pinzón, L. (2013). Transformaciones del modelo neogranadino de parroquialización: el caso de la parroquia San Francisco Xavier de Piedecuesta. Anuario de Historia Regional y de las Fronteras, 18(2), 293-320.

Prada, A. (1995). Piedecuesta: pasado y presente. Piedecuesta: Alcaldía Municipal de Piedecuesta Imprenta Departamental.

Programa Gen-Ondas-Unab (2016). Proyecto "Leyendas y seres legendarios del sector rural de Piedecuesta" a cargo del Semillero Monteredondo. Bucaramanga: Universidad Autónoma de Bucaramanga- Instituto Valle del Río de Oro.

Riveros, D. (2010). Los derechos económicos, sociales y culturales como derechos subjetivos: una visión estructural. Revista Derecho del Estado, 24(1), 29-44

Sanabria, A. (2010). Los derechos culturales en Colombia. Bogotá. Secretaría Distrital de Cultura, Recreación y Deportes.

Uprimny, R. (2016). Los derechos culturales han sufrido de un subdesarrollo jurídico. Revista Arcadia. Disponible en: https://goo.gl/n858ZB

Valenzuela, G. (1995). Piedecuesta: suelo y cielo de Santander. Alcaldía Municipal de Piedecuesta - Imprenta Departamental. 